\title{
ACCENTI CRITICI E PROGETTUALITÀ FORMATIVE PER LE DONNE IN A SERIOUS PROPOSAL TO THE LADIES DI MARY ASTELL (1694) ${ }^{1}$
}

\author{
Antonella Cagnolati ${ }^{2}$ y Sergio Marín Conejo ${ }^{3}$
}

\begin{abstract}
Accenti critici e progettualità formative per le donne in A Serious Proposal to the Ladies di Mary Astell (1694)

Riassunto: Nell'Inghilterra del secolo XVII si andavano argomentando con sempre maggior frequenza nuove proposte per l"educazione femminile, in parte sostenute dalle istanze religiose della Riforma protestante che vedeva nella donna il pilastro morale della famiglia e la prima educatrice dei figli, in parte su esplicita richiesta delle nuove classi borghesi, intenzionate a dare alle figlie una solida istruzione. Le teorie tradizionali elaborate da intellettuali, filosofi e predicatori furono fortemente messe in crisi allorquando le donne si impadronirono della cultura e cominciarono a scrivere trattati in cui rivendicavano per loro i medesimi percorsi educativi previsti per gli uomini, sostenendo che la fonte principale della disuguaglianza risiede nella diversa istruzione che viene impartita, non nel mero aspetto biologico o fisiologico. Il primo trattato pedagogico femminista fu opera di Mary Astell.
\end{abstract}

Parole chiave: educazione, istruzione, pedagogia, femminismo, Mary Astell, Inghilterra, Riforma protestante, trattati educativi.

Critical aspects and educational projects for women in A Serious Proposal to the Ladies by Mary Astell (1694)

Abstract: In 17th century England, new proposals about women education were frequently discussed. On the one, the Protestant Reformation emphasized women as the moral pillar of the family and the main educator of the children. On the other hand, the middle class requested a well-structured and founded education for daughters. Traditional theories developed by intellectuals, philosophers and preachers were deeply criticized when women got involved with culture and began to write treatises asking for the same curricula that was elaborated for men. They thought that the main source of inequality was not based on mere biological or physiological aspects but in the different education. The first pedagogic treatise focused on feminist ideas was written by Mary Astell.

Keywords: education, traning, pedagogy, feminism, Mary Astell, England, Protestant Reformation, educational treatises.

\footnotetext{
${ }^{1}$ Data di ricezione: $24 / 08 / 2016$.

Data di accettazione: 03/10/2016.

${ }^{2}$ Professore Associato di Storia della Pedagogia, Dipartimento di Studi Umanistici. Lettere, Beni Culturali, Scienze della Formazione, Università degli Studi di Foggia, Italia; $\bowtie$ antonellacognalati@unifg.it.

3 Dottore di ricerca, membro del gruppo Escritoras y Escrituras, Facoltà di Filologia, Università di Siviglia, Spagna; $\bowtie$ smarin@us.es.
} 
For since GOD has given Women as well as Men intelligent Souls, why should they be forbidden to improve them? Since he has not denied to us the faculty of Thinking, why shou'd we not (at least in gratitude to him) employ our Thoughts on himself their noblest Object, and not unworthily bestow them on Trifles and Gaieties and secular Affairs?

Mary Astell, A Serious Proposal to the Ladies (London, 1694)

\section{Tracce di rinnovamento.}

Tra la fine del XVI secolo e l'inizio del XVII in Inghilterra si sviluppò un intenso dibattito relativo alla possibile edificazione di una specifica identità femminile, in antitesi o comunque palesemente in controtendenza rispetto all'epoca precedente. L'istanza che aveva motivato lo scatenarsi del confronto su una problematica tanto delicata era rappresentata in primo luogo dalla figura carismatica della regina Elisabetta I: le sue innegabili doti ed il notevole prestigio con cui ella aveva saputo ricoprire il suo ruolo di guida della nazione avevano di necessità dato l'avvio ad una interessante riflessione sulle capacità delle donne in un settore così ostico quale quello della politica. Elisabetta (Cfr. Levin 1994) diventò un'icona talmente prestigiosa che indusse ad un contrastato ripensamento, seppur ancora embrionale durante il suo lungo regno, sui modelli femminili universalmente accettati e condivisi fino ad allora, al punto di porre in discussione la specificità delle caratteristiche muliebri, unitamente ad una meditazione più vasta sui potenziali ruoli del gentil sesso in seno all'istituzione ad esse riservata per eccellenza, la famiglia.

Nella realtà più quotidiana, lontano dai palcoscenici della politica, le donne dell'età moderna trascorrevano la loro esistenza percorrendo pedissequamente i rigidi binari elaborati dagli stereotipi correnti, rivestendo i ruoli di figlia, sposa e madre, 
secondo i dettami delle più condivise convenzioni sociali, rigidamente separate dagli aspetti maggiormente devianti e dalle categorie che ogni comunità tendeva a rigettare, per esempio la strega o la prostituta. L'esplicitazione rituale di consolidati paradigmi funzionava all'interno di una statica suddivisione, modellata sulle due linee guida ben distinte di classe e di genere. Se era opportuno per la donna essere inserita in un contesto socio-familiare che definiva il suo status sempre e comunque in base alle sue relazioni con l'uomo, padre o marito che fosse, si evidenziava nel contempo una netta disparità di giudizio sui suoi atteggiamenti secondo l'appartenenza ad una determinata classe sociale. Oggetto di severa riprovazione se venivano manifestati nelle donne dei ceti umili, taluni comportamenti licenziosi o sconvenienti erano differentemente giudicati e talvolta accettati se entravano a far parte dell'habitus di una gentildonna.

In Inghilterra, negli anni successivi alla Riforma, assai diffuso pareva senza ombra di dubbio l'archetipo della good woman. Suffragato in larga misura dai predicatori, un simile modello riprendeva un topos propagandato nella pubblicistica del tempo ovvero l'immagine della "saggia donna" citata nei Proverbi di Salomone (Proverbi, 31, 10-31.), esempio di virtù e di morigerati costumi, abile nell'amministrazione dell'economia domestica e fedele verso il suo sposo. Partendo da questa figura in larga misura idealizzata, diffusa capillarmente attraverso la predicazione domenicale, lo zelo religioso individuò proprio nella donna il fulcro indispensabile per creare e consolidare una pia e devota famiglia; ci si preoccupò dunque di far acquisire alle giovinette delle competenze che non fossero esclusivamente finalizzate alle abilità domestiche ma che spaziassero in un ambito di carattere etico-devozionale. Un concetto in particolare fu ampiamente condiviso: una giovane pia e virtuosa forniva ottime garanzie perché potesse diventare una moglie onesta e una madre premurosa per i suoi bambini. La figura materna veniva dunque ad assumere un ruolo di notevole portata all'interno della famiglia, seppur sempre necessariamente complementare e subordinato rispetto al marito: la donna, spesso stimata come "a House builded for Generation and Gestation" (una casa costruita per generare e partorire) (Purchas 1619: 474), doveva mostrarsi tenera e amorevole, capace di trasmettere le sue positive qualità ai figli perché crescessero sani ed onesti; rafforzandosi attraverso la lettura di alcuni passi della Bibbia, la figura della buona madre sarebbe 
diventata sempre più fondamentale nelle opere dell"epoca, volte in particolare all'edificazione di una vita familiare morigerata e felice che fosse modellata su saldi principi morali in modo tale da costituire la prima cellula della società. Tuttavia sia le teorie accademiche che le credenze popolari tendevano in ugual misura a vedere la donna come "altro", in relazione all'uomo, riconfermando (se pur ve ne fosse stato bisogno) la necessità di una sudditanza netta e pervasiva nei confronti dei padri e dei mariti: sia il carattere profondamente misogino della società, sia l'impronta patriarcale delle istituzioni necessitavano di un dominio gerarchico ed esclusivo che relegasse la donna in secondo piano. Non di meno, era talmente forte l'influenza delle convenzionali immagini sul gentil sesso che le donne stesse imparavano a pensare in termini di netta differenziazione sessuale:

God made two great Lights, the one to Rule the day, the other the Night: So Man is made to Govern Commonwealths, and Women their private Families... and if it be as Philosophers hold, that the Moon hath no Light but what it borrows from the Sun, so women have strength nor light of Undestanding, but what is given them from Men. (Cavendish 1655, "the Preface to the Reader")

Di fronte ad una ricognizione che poteva in qualche modo minare i capisaldi della società seicentesca incrinando il riconoscimento del dominio maschile all'interno della famiglia e l'indiscussa subordinazione della donna, i detrattori che intendevano relegare le donne in ambiti viepiù ristretti e vincolanti non mancavano: una vasta letteratura tendeva a guardare con smaccata ironia anche il più insignificante tentativo di ipotizzare una possibile uguaglianza tra uomo e donna; nell'atto di definire ed esaltare le virtù femminili, con un'efficace opera di straniamento, si delineavano in realtà i pericoli insiti in un'operazione culturale di tal genere.

Il fattore che più coinvolse gli intellettuali sensibili al problema della rivendicazione di un ruolo maggiormente circoscritto ma in qualche misura impostato su regole differenti fu la religione. Il fervore con cui sia gli Anglicani che i Puritani tentarono di delineare una precisa figura femminile che corrispondesse alle esigenze della virtue and pietie rinveniva sufficienti argomentazioni nella Bibbia, anche se passi di stretta risonanza misógina (Ecclesiaste, 7, 26-28b; Genesi, 3, 1-9) sottolineavano 
l'accondiscendente sudditanza della donna verso le lusinghe del Male, oppure affermavano l'assoluta necessità del dominio del marito sulla moglie: "Le donne siano soggette ai loro mariti come al Signore, poiché l'uomo è capo della donna come anche il Cristo è capo della chiesa, lui, il salvatore del corpo. Or come la chiesa è soggetta al Cristo, così anche le donne ai loro mariti in tutto" (Lettera agli Efesini, 5, 21-24). Lo sesso Pietro, pur ribadendo nella Prima Lettera l'importanza della figura femminile ed il suo ruolo all'interno della comunità cristiana, confermava la sua nostalgia per un tempo lontano quando "le donne sante che speravano in Dio si adornavano e vivevano sottomesse ai propri mariti, come Sara che obbedì ad Abramo chiamandolo Signore" (Prima Lettera, 3, 5-6.). Si palesava la necessità di rendere espliciti ed inequivocabili alcuni elementari principi di carattere etico per controbilanciare la desolante constatazione della perdita di talune categorie basilari volte all'orientamento morale delle donne, considerando come una sciagura l'inevitabile compromissione che le aveva avviate (secondo un'opinione assai diffusa) irrimediabilmente verso la china della degradazione e dell'abbruttimento.

Una seconda icona stava per sopravanzare la figura della regina Elisabetta (modello troppo compromesso politicamente) nell'immaginario collettivo dei ceti sociali impegnati sul fronte religioso: la donna olandese, devota, educatrice ammirevole per la sua prole, aiuto indispensabile per il marito nella gestione dei suoi affari; a tale immagine si riallacciarono coloro che intendevano esaltare le virtù di stampo calvinista, evidenziando come fosse altresì indispensabile educare le fanciulle perché da adulte si avvicinassero il più possibile ad un simile paradigma. Sortisce qui la conseguenza di maggior respiro per la storia dell'educazione: l'affermazione che fosse necessaria un'istruzione che non fornisse abilità soltanto nel cucito e nel ricamo ma che ponesse le donne in grado di leggere ${ }^{4}$ le Sacre Scritture, veicolo di esempi morali da eguagliare. Una serie di opere pubblicate nei primi decenni del XVII secolo ci garantisce un adeguato approfondimento su un'ampia panoramica delle ipotesi più accreditate volte alla ridefinizione del ruolo femminile: in tutte è fortemente presente l'idea che l'istruzione

\footnotetext{
${ }^{4}$ Alcuni testi pubblicati alla fine del XVI secolo propagandavano l'idea che alla donna fosse consentito l'accesso alle scuole per imparare a leggere (ma non a scrivere) (cfr. Mulcaster 1581, W. Kempe 1588).
} 
costituisca un utile strumento per il progresso delle virtù religiose, a patto che fossero posti limiti severi alle potenzialità che una pur limitata alfabetizzazione avrebbe in qualche modo potuto suscitare nell' animo femminile ${ }^{5}$.

\section{Ripensare l'educazione delle donne}

Se un tentativo vi fu per mettere in discussione tali topoi negativi accumulatisi nel corso dei secoli, questo venne attuato nel Rinascimento. Un profluvio di trattati, operette, manuali vide la luce a partire dal Quattrocento, dapprima in Italia e poi, con la progressiva diffusione delle teorie umanistiche, anche nel resto d'Europa (Cfr. Kelso 1978: 326-473).

Sotto il profilo di un miglioramento relativo alla visione della donna, la negatività di tale produzione appare chiara, se consideriamo i contenuti ed il modo in cui si guarda alla figura femminile:

[l'aver visto quel libro], per quanto assolutamente non autorevole, suscitò in me una riflessione che mi turbò profondamente, sui motivi e le cause per cui tanti uomini diversi tra loro per condizione, i chierici come gli altri, erano stati ed erano ancora così propensi a dire $\mathrm{e}$ a scrivere nei loro trattati tante diavolerie e maldicenze sulle donne e la loro condizione ${ }^{6}$.

Il lamento doloroso di Christine de Pizan risulta ampiamente suffragato dalle affermazioni delle maggiori auctoritates più attente e consapevoli del Rinascimento europeo che si dedicano all' analisi dei comportamenti femminili ed alla rigorosa codifica dei comportamenti "corretti” ed auspicabili per il gentil sesso.

Lo sguardo dell'uomo rinascimentale si dirige con minor veemenza sulla riprovazione delle devianti e tenta di plasmare in toto la vita della donna, ben comprendendo che l'educazione gioca un ruolo di enorme rilevanza nella complessa

\footnotetext{
${ }^{5}$ Una tendenza che si raccordava all'apologetica cristiana opponeva all'affermazione che la conoscenza potesse rendere migliore la fede la tesi secondo la quale la capacità di leggere testi latini e greci avrebbe consentito alle donne l'accesso alle licenziose storie che narravano le peccaminose relazioni delle divinità greche e romane.

${ }^{6}$ Faccio riferimento al testo di Christine de Pizan, La città delle dame (1997: 43).
} 
edificazione di un'identità femminile che non sia soltanto complementare al maschile, ma che interiorizzi la consapevolezza che tale modello appare come l'unico possibile e praticabile per la donna dell'época (Cfr. Maclean 1980).

In un saggio di straordinaria valenza nel dibattito storiografico degli anni Ottanta, Joan Kelly ${ }^{6}$ si domandava se per la donna fosse realmente esistito il Rinascimento, intendendo porre come domanda polemica la reale possibilità che tale movimento culturale avesse segnato concretamente un mutamento epocale per la sfera femminile così come si era verificato per l'ambito maschile, determinando l'affrancamento da gerarchie e subordinazioni secolari nel tentativo di affermare la dignità umana. Dunque, affermava Kelly, le risultanze culturali del Rinascimento avevano sempre e comunque privilegiato un'ottica di genere maschile, mentre poche e scarse si erano rivelate le "conquiste" a vantaggio delle donne.

Il pessimismo del saggio di Kelly va in parte corretto e temperato: non si tratta di prendere in considerazione meramente le acquisizioni che il Rinascimento ha attribuito all'uomo quanto di non disconoscere che anche per le donne qualcosa era pur cambiato. Ciò che va rivisto non è tanto la categoria dicotomica maschile/femminile quanto la prospettiva di classe: è indubbio infatti che, se nella maggioranza la popolazione femminile risultava analfabeta, le donne dei ceti superiori ebbero l'opportunità di garantirsi una cultura che, pur circoscritta ad ambiti specifici ed attentamente guidata dall'alto (dal padre, dal precettore o dal sacerdote), le poneva in grado di leggere e scrivere, mettendo in atto tutta una serie di strategie per la comprensione del mondo che ruotava intorno a loro.

Non sarebbe possibile esaltare il prestigio di alcune figure forti di sante e regine senza sottolineare il livello di cultura che tali rappresentanti del sesso femminile avevano potuto acquisire: la presenza di libri nelle loro case, la cura sollecita che ponevano

\footnotetext{
6 "One of the tasks of women'shistory is to call into question accepted schemes of periodization. To take the emancipation of women as a vantage point is to discover that events that further the historical development of men, liberating them from natural, social, or ideological constraints, have quite different, even opposite, effects upon women. The Renaissance is a good case in point" (Kelly-Gadol 1987: 176).
} 
nell'educazione dei propri figli, la volontà di affidare alla carta le loro memorie spirituali o le autobiografie.

Già Erasmo aveva evidenziato un "nuovo" atteggiamento, presentando nei Colloquia alcune figure di donne particolarmente interessanti nel panorama sociale del tempo. Magdalia, protagonista del dialogo Abbatis et eruditae (Erasmo da Rotterdam 2002: 600-611), enfatizza una serie di elementi che senza dubbio Erasmo aveva acutamente osservato probabilmente nella dimora di Tommaso Moro di cui era stato ospite: le figlie di Moro erano l'esempio vivente di una piccola comunità femminile dedita alla cultura, alla devozione religiosa, alla rigorosa osservanza di codici etici di grande valore $^{7}$. Pur nell'ambito di una forte polemica contro l'ignoranza dei monaci, Erasmo tenta di abbattere uno stereotipo millenario constatando una realtà per lui evidente: Magdalia rappresenta un nuovo modello comportamentale, in bilico tra due mondi, il mondo dello status quo, che relega le donne in una sfera privata, escludendole dalla comunicazione culturale e da qualsiasi attività fuori dalle mura domestiche, ed un panorama che vede sgretolarsi il paradigma prevalente ma non scorge ancora un atteggiamento in fieri, considerate le categorie culturali e sociali prevalenti. Magdalia ci pare però una femminista ante litteram quando con toni decisamente polemici sostiene l'assoluta necessità dell'istruzione femminile, considerata la complessità dei ruoli che le donne devono ricoprire.

Tornando al panorama italiano, paiono acquisizioni importanti tutte le riflessioni che l'Umanesimo aveva elaborato sotto il profilo pedagogico: Guarino, Vittorino, Barbaro, Alberti (Cfr. Garin 1958), pur non ponendosi come finalità precipua della loro disamina l'edificazione di un percorso educativo per le fanciulle, toccano seppur marginalmente l'argomento ma è nell'apporto di originalità fornito alla pedagogia stessa che possiamo ritrovare il loro più alto contributo ${ }^{8}$.

\footnotetext{
${ }^{7} \mathrm{Si}$ legga la seguente affermazione: "in Spagna, in Italia ci sono non poche donne nobilissime che potrebbero competere con qualsiasi uomo. In Inghilterra ci sono le donne della famiglia Moro" (Erasmo Da Rotterdam 2002: 609).

${ }^{8}$ Per un'analisi dei testi rinascimentali sulle donne si veda: Bochi (1961) e Lenzi (1982).
} 
Dunque se il panorama europeo si va progressivamente modificando, seguendo le risultanze dell'Umanesimo prima e del più maturo Rinascimento poi, il progetto per un'educazione "femminile" non appare così controcorrente e tende vieppiù ad ipostatizzarsi in un ambito specifico in cui troviamo testi quanto mai diversi tra loro quali libri di devozione, manuali per il buon governo della casa, volumetti per insegnare le buone maniere, trattati che mirano a diffondere i valori della virtù e della castità.

A partire dall'influente opera di J.L. Vives, dedicata a Mary Tudor e tradotta, letta e commentata in tutta Europa, un profondo ripensamento sui topoi più comuni relativi ai percorsi educativi rivolti alle fanciulle si era ormai avviato. Se dal punto di vista teorico permaneva l'atavica e stereotipica concezione della donna come sesso debole e "porta del demonio" 9 , tramandata con veemenza dalla Patristica e rafforzata durante l'arco cronologico del Medioevo, l'idea di un corpo tentatore e pericoloso per le virtù maschili (Maclean 1980), timidi spiragli di rinnovamento stavano apparendo, seppur per le donne che appartenevano ai ceti aristocratici: sia Castiglione ne Il Cortigiano, sia Erasmo da Rotterdam, si erano eretti a paladini di un modello femminile in piena divergenza rispetto al passato, auspicando l'interiorizzazione di una cultura che rendesse le donne più consapevoli del loro ruolo nella società e che prevedesse una alfabetizzazione che le ponesse in gradi di leggere i classici, reperendo altresì nei capolavoro della letteratura antica e cristiana figure ed exempla da imitare per virtù e mirabile devozione. Tale processo, timidamente sbocciato agli albori del secolo XVI, vide pienamente la luce nel Rinascimento, allorquando si moltiplicarono le figure che incarnavano il tipo esemplare pienamente codificato dalla trattatistica pedagogica rivolta alle fanciulle: erano in particolare principesse e regine che, estremamente colte e dotte, consacravano e legittimavano l'acquisizione delle capacità di lettura, di scrittura, l'apprendimento delle lingue classiche e moderne, la dimestichezza nell'amabile conversazione.

Per centrare la nostra disamina sull'Inghilterra, fin dagli albori del regno di Giacomo I Stuart la consapevolezza di una stretta connessione tra la religione e l'educazione era un dato di fatto ormai acquisito: alcuni scrittori insistevano in modo

\footnotetext{
${ }^{9}$ L'espressione è tratta da Tertulliano, De cultu foeminarum (Gli ornamenti delle donne).
} 
particolare sul dovere, da parte dei genitori, di impartire una solida istruzione ai loro figli, senza considerare in maniera pregiudizievole il loro sesso o la loro condizione sociale. Nel suo Of Domesticall Duties, William Gouge conia per la prima volta un binomio che risulterà di grande efficacia durante l'epoca del Protettorato di Cromwell, ovvero l'unione di learning and pietie, ribadendo che il sapere, l'educazione e la vocazione da soli non rivestono un grande significato, se non sono congiunti ad un atteggiamento devoto e compassionevole verso il prossimo: "learning, ciuilitie, calling, portion, are nothing without pietie" (Gouge 1622: 537) ("il sapere, le buone maniere, la vocazione, la dote sono da considerare inutili senza la devozione"). I genitori, raccomanda con solerte sollecitudine Gouge, devono dunque farsi carico di insegnare ai loro bambini i valori morali leggendo loro passi della Bibbia accuratamente scelti per tale scopo ${ }^{10}$. In Inghilterra la richiesta di una maggiore diffusione di scuole da parte della borghesia, classe in costante ascesa sia durante il regno di Elisabetta I che nel secolo successivo, determinò effetti di natura decisamente diversa: se la middle class pretendeva una istruzione che fosse più congeniale alle sue esigenze di tipo "tecnico", lo stesso ceto sociale (ed in primo luogo la gentry) si fece sostenitore delle teorie religiose predicate da Calvino e, come fervido seguace degli ideali puritani, cercò di coniugare la duplice istanza che vedeva nell'educazione una serie di competenze utili per l'inserimento nel mondo del lavoro con la fiducia in una conoscenza più profonda e motivante dei dettami della fede religiosa.

Sebbene l'età in cui si dava inizio ad un percorso educativo fosse la stessa, fra i cinque e i sei anni, senza alcuna distinzione tra maschi e femmine, si riteneva prassi comune approntare un curriculum necessariamente più approfondito, sviluppato lungo un arco cronologico di sette-otto anni per i figli maschi, basato in primo luogo sull'istruzione di stampo classico che avrebbe loro schiuso le porte per luminose carriere nell'amministrazione dello Stato e nelle alte sfere ecclesiastiche; si accedeva così ad una

\footnotetext{
${ }^{10}$ Interessante notare come Gouge criticasse aspramente l'atteggiamento di quei genitori che consideravano l'istruzione un elemento assolutamente superfluo. Sia i bambini nati in famiglie ricche che quelli dei ceti più poveri avevano necessità di acquisire un'efficace educazione: i primi perché grazie alle loro più approfondite conoscenze avrebbero mantenuto o ampliato l'eredità lasciata loro dai genitori; i secondi per potersi procacciare un lavoro che consentisse loro di guadagnarsi da vivere in maniera onesta (Gouge 1622: 529-533).
} 
cultura esplicitamente rivolta verso l'esterno, che avrebbe trovato i suoi pilastri fondamentali prima nel precettore e poi nella rigida disciplina del collegio, una volta che l'allievo si fosse dimostrato idoneo a seguire ed apprendere argomenti più difficili al di fuori della dimora paterna. Una giovinetta di buona famiglia riceveva invece i primi rudimenti della sua istruzione all'interno della sua casa, venendo di norma affidata alle cure della madre, di una governante o di un insegnante che faceva lezione a domicilio. Un pregiudizio analogo può essere rilevato per il termine ad quem in cui collocare la fine dell'iter educativo per le giovani: dal momento che il percorso formativo era finalizzato ad acquisire le competenze necessarie per diventare una buona moglie, si può facilmente dedurre che l'educazione di una ragazza avesse il suo termine fisiologico proprio con il matrimonio: è ragionevole supporre che, data la precoce età in cui venivano celebrate le nozze, tale termine potesse oscillare tra i tredici-quattordici e i diciotto anni.

Che tipo di istruzione ricevevano queste fanciulle? Pare senza ombra di dubbio questo il terreno in cui si riscontravano le differenze più eclatanti. Mentre per i maschi il curriculum studiorum si articolava sul latino, il greco, più raramente l'ebraico, le ragazze imparavano a leggere e a scrivere in inglese, a parlare il francese, a cantare, a suonare uno strumento, a ricamare: il raggio d'azione della pedagogia femminile pare come si vede assai limitato. Nella letteratura dell'epoca, in cui ampio spazio viene riservato all'esaltazione del matrimonio e al ruolo della moglie, la donna deve essere innanzi tutto "una buona padrona di casa, indipendentemente dal fatto che sia di buona estrazione o ricca. Perché tale deve essere la condizione della donna: questo è il suo destino, il motivo per cui è stata creata" (Cfr. Gataker 1624: 173); dunque, mentre una parte della borghesia premeva per l'istituzione di boarding-schools, le istanze religiose andavano definendo una diversa linea di condotta che intendeva fare della donna non un grazioso oggetto con cui trastullarsi bensì una moglie virtuosa e devota, una buona madre e una saggia educatrice per i propri figli.

Tuttavia già dai primi anni del secolo XVII si avanzava da più parti un'immagine dinamica della donna, inserita nella comunità in cui aveva fatto il suo ingresso con il matrimonio e pienamente consapevole dei suoi doveri che venivano esercitati con cognizioni nettamente superiori rispetto al passato: un'ampia trattatistica, così come 
un'abbondante quantità di sermoni, tendevano a fornire nozioni sul buon andamento della casa, sulle cure per preservare i bambini dalle malattie, sul corretto comportamento da tenere nelle occasioni pubbliche, quali le funzioni religiose, le feste e le celebrazioni; l'household government diventa il soggetto preferito sul quale dissertare con dovizia di particolari ${ }^{11}$. Tutte queste fonti hanno in comune il fatto di essere prodotte da uomini che naturalmente osservavano le rappresentanti del gentil sesso attraverso una lente che rifrangeva e spesso distorceva i loro comportamenti, analizzandoli attraverso una rete di pregiudizi misogini, ma nel XVII secolo abbiamo per la prima volta la grande opportunità di ascoltare anche le opinioni delle donne: i loro punti di vista vengono più frequentemente espressi attraverso biografie o epistolari, raramente sotto la forma del trattato. Emergono dagli scritti due opinioni differenti seppur complementari: in primo luogo si riconferma che le donne necessitano di un'istruzione diversa rispetto ai loro mariti o fratelli, dal momento che un eccesso di cultura potrebbe rivelarsi alla lunga uno svantaggio sotto il profilo sociale, per esempio nella ricerca di un buon partito matrimoniale. La seconda tesi ribadisce che la totale mancanza o la carenza di istruzione nelle donne è comunque un danno che si riflette sulla famiglia e sull'intera società: considerando il futuro ruolo di educatrici dei propri figli, si esprime da più parti la perplessità che madri scarsamente istruite siano realmente in grado di educare al meglio la loro prole sulla via della cristianità.

Educate a casa o nelle scuole per signorine, le donne cominciarono a scrivere: inizialmente si trattava di semplici diari religiosi, manuali di preghiere e testamenti spirituali, che sfociarono in un largo numero di pamphlet relativi a problemi sociali riletti adottando un'ottica devozionale. Le pubblicazioni stimolarono gli scrittori a cimentarsi nello stesso genere attraverso opere che risultavano essere una semplice denuncia in forma oratoria oppure erano incentrate su tematiche educative come, per esempio, The Ladies Calling (Allestre 1673). Sebbene Allestre non argomenti in favore di una parità intellettuale tra uomo e donna, egli afferma che le donne sono in grado di progredire mentalmente e che la loro intelligenza diventa debole a causa dello scarso utilizzo.

\footnotetext{
${ }^{11}$ Come esempi di tale trattatistica si possono citare Christian Oeconomy, or Household Government di F. Dillingham oppure A Godly Form of Householde Government di R. Cleaver.
} 
Riconosce che le donne, essendo dotate di un'anima, se utilizzassero un comportamento adeguato, potrebbero meritare il regno dei cieli. Egli presenta le caratteristiche ideali di una donna, nonché una completa descrizione degli stadi attraverso cui questa passa durante la propria esistenza: verginità, matrimonio e vedovanza, sottolineando come alla donna debba essere opportunamente riservato l'insegnamento della cura della casa, il vestirsi sobriamente, lo scrivere, il cantare e ballare, come debba seguire gli usuali comandamenti morali, quali la castità e il silenzio all'interno della chiesa. Si ribadisce l'antico concetto secondo cui uomo e donna sono stati creati l'una per obbedire ai voleri dell'altro essendo la natura femminile subordinata: durante la sua vita la donna è sempre soggetta ad una autorità, quando è una bimba ai genitori, quando è sposa al marito, e quando rimane vedova a Dio. L'autore si lamenta per la mancanza di conventi in Inghilterra che hanno diminuito la possibilità per le donne di servire Dio nella maniera più corretta, e nello stesso tempo non può che ammirare quelle ragazze che decidono di donare la propria vita al servizio dell'Altissimo rifiutando il matrimonio e mantenendo una pia condotta per tutta la loro esistenza. Coloro che si sposano devono essere indirizzate al matrimonio dai propri genitori i quali sceglieranno colui che ritengono il compagno migliore.

Nello stesso periodo venne pubblicato The Lady's New-year's Gift: or, Advice to a Daughter (Saville 1688) del marchese Halifax, opera nella quale vengono rappresentati i compiti della donna e i suoi ineludibili doveri. Il testo, dai toni molto più informali, è concepito come un manuale pratico che il marchese scrive alla propria figlia per aiutarla a superare nel migliore dei modi le avversità della vita, sottolineando più volte che alle donne è riservato un ruolo subordinato rispetto agli uomini. Queste due opere, nonché le teorie e le prassi che suggerivano, ispirarono la successiva discussione sulla posizione del weaker sex all'interno della società al punto che la loro influenza fu sostanziale per ribadire concezioni misogine e patriarcali, secondo cui la donna è una creatura naturalmente subordinata sia fisicamente che intellettualmente all'uomo (Smith 1982: 46).

Tuttavia pur lentamente si registravano cambiamenti. La Riforma in primis aveva sollecitato in eguale misura uomini e donne al libero esame, alla lettura e 
all'interpretazione individuale della scrittura. Le confessioni protestanti, alle quali aderivano in maggioranza le donne, sostenevano l'uguaglianza spirituale tra i generi e chiedevano per le donne un ruolo più attivo all'interno della congregazione religiosa. Sebbene il patriarcato fosse ancora solido, all'interno delle chiese riformate alle donne era concessa maggiore libertà espressiva, in quanto illuminate da Dio. La Guerra civile aveva rafforzato tali spinte egualitarie all'interno della società e fu proprio in questo periodo che iniziò a formarsi la consapevolezza di un'identità femminile e una partecipazione più attiva alla sfera pubblica. Tale fermento liberale fu arrestato dalla Restaurazione degli Stuart nel 1688 e dagli ideali negativi che essa promuoveva. Vennero riprese ed esaltate le argomentazioni misogine che da secoli condizionavano la donna e la sua identità, come le Lettere ai Corinzi ed il mito di Eva. Proliferarono i libelli che sostenevano l'inferiorità morale e cognitiva del gentil sesso e denigravano le femmes savantes, considerate stupide e pericolose, capaci con i loro discorsi solo di mettere in difficoltà la tranquillità familiare e sociale (Thomas 1958: 42-62).

Il parziale ritorno all'antico non pose fine alle discussioni sulla posizione sociale ed intellettuale della donna: le concezioni cartesiane permettevano di riformulare, con una più consistente criticità e coerenza argomentativa, le teorie sull'uguaglianza dei sessi. Il rifiuto di accettare la tradizione senza prima adeguate verifiche, la diffidenza nei confronti del consenso sociale, la garanzia di pervenire ad una conoscenza mediante l'utilizzo della ragione pensata come facoltà umana autonoma, permisero l'elaborazione di una nuova visione della donna. La diversità che divideva i sessi non veniva più imputata alla natura, ma al contesto culturale, ai comportamenti codificati dalla tradizione e alla disparità di educazione: l'esigenza di un'istruzione migliore per la donna acquistò nuovo significato e venne sostenuta da più forti argomentazioni.

Per abbattere dunque in maniera efficace il muro di pregiudizi costruito intorno all'ipotesi di un percorso culturale costruito esplicitamente sulle esigenze delle donne, occorreva una decisa presa di posizione, volta dapprima a negare l'inferiorità del gentil sesso, in aperta polemica con i difensori di tale tesi, e ad affermare poi una sostanziale identità dell'uomo e della donna nei confronti delle loro potenzialità educative. Proprio muovendosi lungo tale direttrice, alcune scrittrici inglesi dovettero innanzi tutto ridefinire 
il ruolo della donna nel campo dell'istruzione, esprimendo una posizione pedagogica innovativa che si allontanava dalla tradizione consolidata, per rivalutare pienamente la figura femminile sotto il punto di vista che era stato da sempre ampiamente sottovalutato, se non recisamente negato: la capacità di utilizzare l'intelligenza per migliorare la propria vita. La più fervente sostenitrice di tale dirompente prospettiva fu Mary Astell (16661731).

\section{La modernità della proposta educativa di Mary Astell}

Spinta dal suo mentore John Norris che la sosteneva apertamente esaltando il suo spiccato ingegno e le sue profonde doti intellettuali, Mary Astell ${ }^{12}$ pubblicò nel 1694 la sua personale proposta educativa. Suo più grande desiderio consisteva nel distogliere le menti delle sue contemporanee dalle frivolezze della mondanità, dirigendo le loro riflessioni su argomenti più proficui che permettessero loro un diverso approccio alla realtà e una maggiore possibilità di redenzione. Astell decise di utilizzare il genere letterario dell'epistola che le permetteva una maggiore libertà stilistica, allontanava le possibili malignità e critiche, non le imponeva un vocabolario complesso e una rigida struttura formale; in aggiunta, presentandosi come una conversazione tra donne, le consentiva di entrare in immediato contatto con le interlocutrici.

Il Serious Proposal (Astell 1694) è la prima opera pubblicata da Astell. Possiamo solo immaginare quanto sia stato difficile compiere questo passo: ella aveva poco meno di trent'anni, era ancora una giovane donna sconosciuta, viveva solitaria nel sobborgo londinese di Chelsea tentando di sopravvivere con la carità della curia vescovile e di qualche mecenate. Sebbene la sua fama di donna colta si stesse rapidamente diffondendo nei circoli di corte, pubblicare un'opera poteva rivelarsi rischioso: se il pubblico non avesse apprezzato lo scritto, questo avrebbe certamente attratto critiche e scherno. Mary ben conosceva la vena satirica che in quel tempo si scatenava contro la maggior parte dei testi composti da donne, o che ne difendevano l'integrità morale e spirituale. La sua

\footnotetext{
${ }^{12}$ Per una profilo biografico di Astell si veda l'approfondito studio di Ruth Perry (1986).
} 
credibilità avrebbe subito un irrimediabile crollo e sarebbe stata trattata come l'ennesima femme savante che si impegnava in complicate discussioni che non sempre dimostrava di saper padroneggiare agevolmente. Uscendo dalla sicura sfera del privato la sua reputazione ne sarebbe risultata ineluttabilmente macchiata, impedendole amicizie e rapporti sinceri con le persone più influenti della cittadina nella quale viveva.

Protetta dall'anonimato (Mary si firma a Lover of Her Sex), nonché armata di notevole coraggio, ella decise di avventurarsi nel mondo della stampa, affermando che poco le sarebbe importato di cadere nel ridicolo se con il suo testo fosse stata in grado di aiutare le donne a formarsi una propria consapevolezza:

She [the author] is very indifferent what the critics say, if the Ladies receive any advantage by her attempts to serve them, so it will give her the greatest uneasiness if having prov'd that they are capable of the best things, she can't persuade to a pursuit of them. It were more to her satisfaction, to find her Project condemned as foolish and impertinent, than to find it received with some approbation and yet nobody endeavouring to put it in practice ${ }^{13}$.

La Part I, dedicata alle donne, si articola in vari punti: un'iniziale richiesta di attenzione, in cui si promette di accrescere il fascino delle signore se seguiranno i consigli riportati, segue l'analisi della realtà della donna, la sua degradazione morale e intellettuale, le sue cause profonde. Terminata la pars destruens, Astell passa a descrivere e suggerire i rimedi che si possono adottare per risollevare la condizione delle ragazze con un'istruzione che permetta loro di conseguire autonomia di pensiero vincendo i vizi per avvicinarsi alla virtù. Esplicita poi la concreta proposta pedagogica in cui sono descritte le metodologie dello sviluppo educativo, concludendo circolarmente con un'ulteriore analisi dei benefici che l'istruzione comporta.

Fin dall'esordio ella chiarisce il suo intento: scrive per accrescere il valore interiore delle donne, impedendo che restino meschine e senza scopo, valorizzando la loro purezza interiore piuttosto che l'effimera bellezza esteriore. "In a word, render you

\footnotetext{
${ }^{13}$ Astell (1697). Tutte le citazioni dal testo provengono dall'edizione Astell, A Serious Proposal To the Ladies, parts I e II, edited by Patricia Springborg, London, Pickering and Chatto, 1997 (cit. a p. 119).
} 
the Glory and Blessing of the present Age, and the Admiration and Pattern of the next" (52). L'autrice si propone di far rigettare alle donne l'ideale della vanità loro imposta dal modello maschile, per riportarle all'equilibrata dignità della loro natura che, seppur imperfetta, permette di raggiungere appieno la felicità. Il ritorno alla virtù deve rappresentare lo scopo fondamentale di ogni donna: ciò non significa ritirarsi dalla vita del mondo per rinchiudersi in un eremo, ma evitare di attirare gli sguardi degli uomini, e servire Dio nella maniera più completa. Fin dall'esordio si evince l'aspetto pio e cristiano della personalità di Mary, la determinazione e l'audacia nel condannare il comportamento che gli uomini manifestano nei confronti delle donne: "We're indeed oblig' $\mathrm{d}$ to them for their management, in endeavouring to make us so, who use all the artifice they can to spoil, and deny us the means of improvement" (57) Invece di chiedersi come mai tutte le donne non siano sagge e buone, è più giusto meravigliarsi che ve ne siano di sagge e buone. Gli uomini hanno lasciato le donne in balia di se stesse, trascurando la loro cultura e il loro progresso intellettuale, facendole letteralmente affondare nella stupidità e nell'abbruttimento morale.

Asserita la responsabilità dell'uomo come spiegazione più plausibile del comportamento irrazionale della donna, Mary si appresta a criticare il concetto di inferiorità naturale delle figlie di Eva postulato da uomini arguti e maligni che ne hanno fatto il mezzo per soggiogare e controllare le proprie compagne:

The Incapacity, if there be any, is aquired not natural: [...] The Cause therefore of the defects we labour under, ism if not wholly, yet at least in the first place, to be ascribed to the mistakes of our Education; which like an Error in the first Concoction, spreads its ill Influence throught all our Lives. (59-60)

Sin dall'infanzia, ella continua, viene negato alla donna un'educazione seria e funzionale, la si lascia crescere cullata da vizi che non avrà più possibilità di estirpare una volta adulta. Mary descrive quale debba essere il comportamento che i genitori devono assumere nei confronti dei proprio figli: permettere una vita terrena felice e ottenere poi gioie infinite nell'aldilà con un'educazione mirata. Il dovere di una madre è nobile e fondamentale, a lei sola è data la possibilità di salvare l'anima dei bimbi dalle bruttezze 
del mondo. Deve quindi guardare alla propria discendenza con la dovuta considerazione compiendo appieno l'obbligo naturale dell'allattamento o, se impossibilitata, controllando minuziosamente che la balia non trasmetta con il proprio latte vizi e cattive abitudini ${ }^{14}$.

Secondo Astell, il periodo dell'infanzia risulta fondamentale per il corretto sviluppo delle bambine, ed è da imputare alla mancanza di educazione l'inclinazione futura a prendere decisioni negative. Solo fondando la fede su conoscenze razionali una donna potrà pervenire ad un sincero sentimento di pietà, perché se lasciata allo sbando verrà attratta solo da quegli aspetti futili della vita come la bellezza e il denaro. D'altro canto l'uomo sarà in grado di circuire le giovani, prive di armi per difendersi dalle lusinghe ${ }^{15}$ : "he has the Poor un happy Person at his Mercy, who now very glibly swallows down his Poyson, because "tis presented in a Golden Cup; and credulously hearkens to the most disadvantagious Proposals, because they come attended with a seeming esteem" (Astell 1997: 63). È l'ignoranza che inganna, continua Mary, inducendo a rinunciare alla sostanza per l'ombra, alla realtà per l'apparenza e stimolando ad abbracciare i vizi piuttosto che le virtù. Sebbene alla donna vengano insegnati i rudimenti della religione, non le si permette di conoscerne i fondamenti e le sue ragioni, indebolendo la sua fede che vacillerà alla prima lusinga.

\footnotetext{
${ }^{14} \mathrm{Nel}$ XVII secolo era pressoché impossibile trovare una donna della classe agiata che non ricorresse al baliatico per nutrire il proprio figlio. Risultava impensabile per le signore di nobile lignaggio abbassarsi a compiere un compito così disgustoso come allattare al seno il proprio infante. Le madri dei ceti superiori affidavano i figli ad una balia giustificandosi di non essere in grado di produrre latte in quantità sufficienti, ma in realtà ritenevano l'allattamento un compito del tutto privo di prestigio sociale. I figli venivano affidati alle balie per la convinzione che l'allattamento fosse incompatibile con i rapporti sessuali, perché questi interferivano con la distribuzione del sangue nel corpo della donna, determinando problemi alla qualità e alla quantità del latte e arrivando persino a provocare la morte del feto. Già in questa epoca, comunque, specialmente da parte protestante, si iniziava ad attaccare l'usanza del baliatico, ritenuta disumana e nociva per i bambini. Le argomentazioni più diffuse a difesa dell'allattamento materno si incentravano sul dovere della madre di nutrire il proprio figlio, dovere che era stato imposto da Dio.

${ }^{15}$ Il tema delle lusinghe maschili che fanno ricadere una donna tra le braccia di un uomo senza che essa riesca ad averne una reale consapevolezza verrà ripreso nello scritto Some Reflections Upon Marriage (1700), ed è uno degli argomenti preferiti di Mary che vede nell'educazione non solo il momento in cui si riconosce la parità intellettuale delle donne, ma anche quello in cui una ragazza acquista la piena consapevolezza di sé senza la quale sarà impossibilitata a fare le sue scelte in maniera coerente.
} 
Esposte le cause principali che spiegano i comportamenti frivoli e sciocchi di una donna, Mary affronta la pars construens del suo trattato, nella quale indica i migliori metodi per donare alle fanciulle la migliore educazione possibile:

Now as the Proposal is to erect a Monastery [...] and such as shall have a double aspect, being not only a retreat from the World for those who desire that advantage, but likewise, an institution and previous discipline, to fit us to do the greatest good in it; Such an institution as this [...] would be the most be the most probable method to amend the present and improve the future Age. [...] You are therefore Ladies, invited into a place, where you shall suffer no other confinement, but to be kept out of the road Of Sin, [...] You will only quit the Chat of insignificant people for an ingenious Conversation. (73-74).

L'eremo felice permette alla donna di occuparsi completamente a servire Dio ed accrescere la propria mente lontana dai clamori del mondo, sostenuta dall'affetto sincero delle altre compagne, con la possibilità di innumerevoli discussioni sulle discipline approfondite. Il ritiro consente di unire alle opere buone di una esistenza attiva il piacere e la serenità della vita contemplativa, permettendo alla donna di agire in maniera caritatevole nei confronti della società.

Dopo aver analizzato i vantaggi della sua accademia rispetto alla vita religiosa, Mary definisce i programmi di studio che permettono un reale miglioramento. Innanzitutto devono essere impartiti i fondamenti delle lingue straniere, necessarie per una corretta lettura di tutti i testi scientifici e filosofici. Alle fanciulle verrà quindi insegnato il latino e il francese, indispensabili per l'approccio agli autori più importanti. Non è consigliato leggere un'immensa moltitudine di libri, ma semplicemente comprenderne e assimilarne pochi, validi e accuratamente scelti evitando testi teatrali e romanzi, che non forniscono alcuna conoscenza utile, ma tendono alla speculazione fantastica dannosa per la formazione di una mente razionale. Anche nell'organizzazione della giornata occorre seguire i ritmi monastici, intervallando lo studio con la preghiera. I pasti dovranno essere frugali per permettere una maggiore concentrazione. Le maestre che seguono la cura spirituale di queste fanciulle devono essere persone irreprensibili che abbiano dedicato la loro intera esistenza allo studio e alla pratica del cristianesimo. 
Sebbene le fondamenta di questa scuola siano monastiche, i suoi membri vi entrano solo per apprendere maggiori conoscenze del mondo, e possono uscirne in qualsiasi momento o per sposarsi, o per iniziare delle opere pubbliche di carità. Per questo motivo la scuola è pensata per fanciulle provenienti dalle classi nobili, alle quali devono essere insegnate le nozioni più importanti. Le ragazze provenienti dai ranghi più bassi potranno comunque avere un posto all'interno dell'istituzione, occupandosi dell'economia domestica avendo la fortuna, grazie al contatto con le nobildonne, di imparare, almeno parzialmente, alcune delle più importanti idee che riguardano il mondo. Come ha notato Florence Smith, questa pare la più grave limitazione del pensiero della Astell, non essendo in grado di staccarsi dall'ideale di classe e quindi confinando l'educazione solo all'interno della nobiltà (Smith 1916: 54).

Per comprendere appieno come Mary sia pervenuta all'ideale di ritiro religioso di donne, dobbiamo rifarci alla sua biografia. Alla morte del padre la giovanissima Mary visse per tutta la sua adolescenza in una piccola comunità di donne formata dalla madre, da una vecchia zia e da qualche domestica. La madre, troppo orgogliosa per richiedere alla figlia un aiuto nelle faccende di casa, ma troppo povera per permetterle una vita sociale, aveva consentito alla ragazza di continuare quel curricolo di studi, cui era stata iniziata dallo zio Ralph, senza dimenticare una stretta osservanza della religione. Mary iniziò a strutturare l'idea di una esistenza all'interno di una collettività femminile come alternativa al matrimonio e all'amore grazie a questa sua esperienza:

We shall have the opportunity of contracting the purest and noblest Friendship; a Blessing, the purchase of which were richly worth all the World besides! For she who possesses a worthy Person, has certainly obtain'd the richest Treasure! A Blessing that Monarchs may envy. [...] For Friendship is a Vertue which comprehends all the rest; none being fit for this, who is not adorn'd with every other Vertue. Probably one considerable cause of the degeneracy of the present Age, is the little true Friendship that is to be found in it. (98)

Il ritiro non solo offre l'immensa possibilità di contrarre amicizie vere, ma sottende vantaggi ben più materiali. Può diventare il sospirato rifugio per l'ereditiera stanca di essere rincorsa per il suo denaro, almeno finché i genitori non saranno in grado di trovare per lei la miglior sistemazione matrimoniale possibile; costituire il luogo 
ideale nel quale le famiglie nobili possano educare le proprie figlie affinché diventino il plauso del casato; può divenire l'ancora di salvezza di tutte quelle gentildonne decadute che non hanno una dote che permetta loro un dignitoso matrimonio.

A conclusione di questa prima parte, Mary controbatte le obiezioni mosse dai contemporanei sulla necessità di un'educazione alle fanciulle. A chi afferma che un'istruzione colta rende la donna vana e presuntuosa e che invece di correggere il suo orgoglio tende ad accrescerlo, Mary risponde che ciò può accadere, ma solo a chi ha ricevuto un'istruzione superficiale, a chi si è fermato all'apparenza piuttosto che giungere alla sostanza. Motivo per cui nella sua accademia desidera fornire alle donne un'istruzione seria e profonda che permetta loro di acquisire consapevolezza ed evitare di parlare a sproposito. Gli uomini devono accorgersi come l'istruzione femminile sia a loro completo vantaggio: una madre competente e accorta sarà in grado di plasmare rettamente la mente del suo bambino sollecitando risultati positivi in tutti gli stadi della vita, una moglie ragionevole e prudente sarà di aiuto al marito. Dimostrata la concretezza e la giustezza della sua causa, Mary si appella alla benevolenza dei suoi lettori perché riconoscendo i reali vantaggi della istituzione la finanzino con delle offerte che permettano la sua costruzione; richiesta di sponsorizzazione particolarmente moderna.

\section{Stimoli per l'intelletto femminile}

Nel 1697 fu pubblicata la seconda parte del Serious Proposal che Mary decise di dedicare alla regina Anna di Danimarca, nota per essere una fervente sostenitrice della causa femminile. Sicuramente sperava che la dedica potesse garantirle aiuti finanziari per la costruzione della sua accademia. Se nella parte prima del Serious Proposal aveva dimostrato come la capacità intellettiva fosse identica tra uomini e donne, nella seconda parte Mary focalizza la sua attenzione sui metodi con cui raggiungere reali progressi. Lo scritto è diviso in quattro capitoli: il primo ribadisce le ragioni per cui una donna deve ricevere una perfetta educazione, i restanti mostrano come la conoscenza può essere ricercata attraverso lo studio del funzionamento dell'intelligenza del singolo. 
Le donne devono basare la loro fede sulla ragione, e non sull'imposizione dei padri o dei mariti, per cui è essenziale vengano impartiti quei fondamenti conoscitivi in grado di sviluppare un sincero attaccamento alla chiesa e ai suoi dettami. È necessario seguire il costume finché la ragione non sia perfettamente sviluppata ma una volta raggiunte le conoscenze necessarie, la donna può essere libera di formulare autonomamente le proprie scelte. Mary non spiega se la facoltà di giudizio sia controllata dalla ragione o dal desiderio, il suo intento è infatti dimostrare i difetti del pensiero non addestrato in maniera corretta. Una persona alla quale non sia stato insegnato a seguire la sua razionalità agirà maggiormente assecondando desideri ed istinti, anche se in realtà non potrà completamente mettere a tacere la ragione. "There are some degrees of Knowledge necessary before there can be any Human Acts" (129). Se viene insegnato a questa anima sfortunata a pensare a cose più alte, essa raggiungerà quella purezza di cuore necessaria a compiere azioni in accordo con l'onestà e la virtù.

La sezione che segue analizza le cause dei comportamenti scorretti e le azioni da contrapporvi. Vi sono pigrizia e indifferenza che non permettono una corretta concentrazione su ciò che è realmente importante: l'avvicinarsi quanto più possibile alla verità. Occorre abbandonare tutti i pregiudizi acquisiti con l'educazione o dettati dall'autorità e dal costume "not give credit to any thing any longer because we have once beliv'd it" (133). Il fatto che molti avvallino una nozione, non consente la certezza della sua veridicità. È opportuno indagare il mondo partendo dal presupposto che la verità verrò rivelata solo a coloro che vi si approcciano con cuore puro e mente aperta. Il self love ${ }^{16}$ è lo strumento che, se positivamente utilizzato, permette una notevole apertura mentale e

\footnotetext{
${ }^{16}$ Mary utilizza lo stesso termine reso famoso da Pierre Nicole (1625-1695) nella sua teoria delle passioni che distingue tra l'amour-de-soi, ovvero quel sentimento positivo e sano che accresce la nostra stima personale, e amour prope, che è una forma negativa di amore nei propri confronti poiché tende ad escludere l'altro basandosi sull'orgoglio e sulla vanità. Mary Astell segue perfettamente la distinzione di Nicole e la utilizza per sostenere le proprie argomentazioni. Filosofo e scrittore Pierre Nicole nacque a Chartres, in Francia, nel 1625. Nel 1642 si recò a Parigi per studiare teologia ed entrò ben presto in contatto con l'ambiente del convento di Port-Royal, riferimento giansenista dell'epoca in città. Nicole non prese gli ordini maggiori, rimanendo ad ogni buon conto un teologo di notevole preparazione, ed insegnò per diversi anni letteratura, lingua greca e filosofia nella scuola di Port-Royal. Nel 1662 scrisse, in collaborazione con Antoine Arnauld, La Logique ou l'art de penser (La logica, o arte di pensare), noto anche come La logica di Port-Royal, un testo popolarissimo ai tempi, ristampato ben cinque volte, che trattava temi di logica, linguaggio, teoria della conoscenza e della metafisica. L'opera è suddivisa in quattro parti che prendono in esame le quattro principali operazioni della mente: il concepire, il giudicare, il ragionare e l'ordinare.
} 
un conseguente avvicinamento alla verità, ma se si trasforma in amor proprio preclude qualsiasi possibilità di crescita, trattenendo tutti i pregiudizi e gli errori acquisiti in precedenza. In questo caso accade che invece di cercare nuove argomentazioni per spiegare la verità, costruiamo nuove ipotesi che confermano le nostre precedenti supposizioni, anche se errate.

Adducendo un'affermazione che in parte smentisce le precedenti argomentazioni, Mary si contraddice sostenendo che "do not design any Prejudice to the Authority of the Church which is of the different consideration" (138). Nessuno deve porre in dubbio ciò che è affermato dalla chiesa nazionale, o anche dalle chiese particolari, a meno che i loro dettami non siano completamente opposti a quelli delle Sacre Scritture. Cercando di salvaguardare la sua posizione contro le autorità senza delegittimare il peso della Chiesa, Mary distingue tra due differenti tipologie di verità: la prima di tipo filosofico, che può essere ricercata da tutti ma che solo alcuni possono raggiungere, la seconda di tipo morale che deve essere seguita e raggiunta dalla totalità degli individui attraverso il giusto uso della ragione.

Una "Generous Resolution" unita ad una "Courageous Industry" rappresentano gli unici aspetti che permettono all'uomo di uscire dallo stato di torpore indotto dalla pigrizia e dall'indifferenza e di superare i pregiudizi acquisiti tramite l'educazione e il costume. Coraggio e risoluzione permettono, una volta raggiunte le conoscenze veritiere, di ritenerle con continuità. Per concludere Mary indica quale debba essere lo scopo finale della ricerca razionale. L'intelligenza si deve basare sulla moralità, unica azione consona all'uomo che possa condurlo alla piena felicità.

La terza sezione descrive quali siano le metodologie per perfezionare la conoscenza razionale. Dapprima bisogna studiare le possibilità della mente umana, dal punto di vista generale e da quello particolare; si passa poi a descrivere i più comuni incidenti di comprensione dell'intelligenza e come possano essere superati applicando la logica naturale e la retorica. Infine ella elenca gli usi possibili della conoscenza, applicando tali argomentazioni all'educazione della donna, per dimostrare che vi è un fondamento razionale alla sua istruzione. 
La perfezione della mente umana "consisting in the Clarness and Largness of its view" (144) migliora quando le idee diventano più chiare ed estese. L'obiettivo fondamentale è la ricerca della Verità, raggiungibile con molteplici percorsi, essendo la mente finita e limitata: "this Limitation is the cause of those different Modes of Thinking, which for distinction sake we call Faith, Science and Opinion” (149). Per cui esistono diverse strade per raggiungere la conoscenza. Ogni uomo possiede una propria struttura mentale fondata sulle più profonde inclinazioni che gli permette di raggiungere la verità secondo le capacità personali. Per favorire un uso migliore dell'intelligenza è importante che l'educazione del singolo sia differenziata a seconda delle attitudini.

Per pervenire ad una corretta conoscenza razionale, Mary suggerisce varie tappe derivate quasi testualmente dalle regole del metodo cartesiano ed in grado di dirigere la conoscenza. Innanzitutto occorre avere una distinta nozione del soggetto da indagare, secondariamente scartare tutte le idee che non sono necessarie per lo studio della materia concentrando l'attenzione solo su quelle di cui abbiamo chiara nozione, in terzo luogo condurre il ragionamento con ordine procedendo per stadi di conoscenza successivi, dal più piccolo al più grande, per poi dividere il problema in diverse parti che devono essere perse in considerazione tutte con uguale attenzione, tenere il nostro soggetto costantemente in esame cercando di annotare i progressi, infine evitare di accettare un elemento per vero se non lo si può dimostrare.

Nella parte seguente Mary discute le tecniche di espressione linguistica (logica e retorica) applicate all'educazione della donna. Un'approfondita conoscenza delle regole retoriche è fondamentale per pervenire ad una correttezza di pensiero, così come è fondamentale coltivare nella fanciulla il senso della verità che, unita allo studio retorico, favorisce una notevole correttezza nell'espressione del personale punto di vista. La capacità di parlare di fronte ad una platea non deve essere introdotto nel curricolo di studi della giovane, poiché la donna non verrà mai chiamata a partecipare ad agoni pubblici e la voce che le ha donato la natura è sufficiente per la conversazione.

L'obiettivo dell'educazione risiede nel miglioramento della vita. Una mente razionale in grado di pensare correttamente permette di scegliere la condotta morale 
migliore perché riesce a discernere l'azione più adatta e valutarne le conseguenze all'interno della società. Una donna istruita non solo è in grado di assumere un corretto comportamento, che donerà lustro alla sua casata, ma possiede tutte quelle capacità necessarie per compiere al meglio il suo più grande dovere: la cura e l'educazione dei figli:

Education of Children is a most necessary Employment, perhaps the chief of those who have any; But it is as Difficult as it is Excellent when well perform'd; and I question not but that the mistakes which are made in it, are a principal Cause of that Folly and Vice, which is much complain'd of and so little mended. (202).

Il successo o l'insuccesso nella cura dei bimbi viene quindi fatto ricadere sulla madre, specialmente se il capofamiglia è impegnato nelle sue attività. L'educazione del bambino non richiede solo tenerezza e cura, ma una quantità di conoscenze che evitino gli errori più grossolani. È importante educare anche una fanciulla non sposata, perché sia in grado di discernere le menzogne dalla verità e di scegliere consapevolmente il proprio sposo.

Il capitolo conclusivo si focalizza sul controllo dei desideri e sul buon governo delle passioni in accordo con le conoscenze acquisite. Per Astell, la natura umana ha in sé il desiderio, importante passione che spinge l'uomo alla ricerca della felicità terrena. Mary non nega la giustezza di questo moto dell'anima, ma si chiede come sia possibile che la maggior parte degli individui preferisca appagarsi con il limitato, ma immediato piacere che offrono i peccati, piuttosto che affidare la propria esistenza a Dio, unico in grado di donare la felicità infinita ed eterna. Per rendere ragione di questo comportamento, l'autrice riflette sulla natura umana e sulle passioni dell'anima: "it is thro the mistake of some of these that our Inclinations take a wrong bias, and consequently that we transgress against God, our Neighbour and our selves" (210). Come Cartesio prima di lei, Mary Astell fa originare le passioni da certi movimenti del sangue e dagli spiriti primordiali che spesso occludono la mente non permettendole di funzionare razionalmente. I sensi tuttavia non devono essere condannati poiché sono stati dati da Dio per farci provare piacere e per guidarci, ma devono essere tenuti sotto controllo evitando di stimolarli eccessivamente. 
Le conclusioni del capitolo ribadiscono la parità intellettuale delle donne messe al mondo per fare il miglior bene possibile. Mary sprona le donne ad avere un'alta considerazione di sé e ad utilizzare un'accorta riflessione per non cadere in quella mediocrità che la società auspica per loro.

Come ha notato Ruth Perry (1986: 100), non è difficile comprendere la popolarità di A Serious Proposal: le due parti sono ironiche, briose e ricche di sensibilità. La part I è probabilmente l'unità meglio riuscita: Mary vi palesa tutta la sua passione di paladina delle donne, polemizzando aspramente, arguendo con impeto e combattendo con fervore. In questo primo segmento esprime liberamente i sentimenti; contrariamente, nella seconda parte ricalca la scrittura ideologica del suo tempo, esponendo una dottrina filosofica praticamente identica a quella elaborata dai suoi contemporanei. La prima parte è libera da tutti quei pregiudizi che per secoli si sono accumulati soffocando la donna e la sua intelligenza, il materiale presentato è piuttosto innovativo e concreto, viene ribadita l'uguaglianza di genere e fornita la descrizione di un luogo e un curricolo di studi che assicurino la reale uguaglianza intellettuale; la seconda descrive il metodo con cui accrescere le capacità intellettive e permettere il raggiungimento dell'eterna felicità, "followed in large measure the Cartesian philosophy" (Smith 1916: 60). Il contenuto appare da subito poco originale, vi si ritrovano combinate le teorie della conoscenza maggiormente in voga alle quali non viene aggiunto alcun contributo personale. Mary si basa sulla logica della scuola di Port-Royal, quando analizza l'importanza della ricerca della verità e descrive l'identità del sé, su Cartesio quando enuncia il suo personale metodo di conoscenza e ricorre ulteriormente al filosofo francese per sostenere la sua teoria delle passioni. Il suo contributo alla storia dell'educazione non può essere ritrovato in teorie scientifiche originali "but in her practical application of her ideas and in her clear view of the social possibilities lying back of the education of the women" (61). Una donna se istruita sarà maggiormente produttiva all'interno della casa, educherà i propri figli rettamente e facendone uomini migliori, eviterà una scelta irragionevole unendosi in matrimonio con maggiore consapevolezza e quindi con maggiori possibilità di essere felice. 


\section{Riferimenti bibliografici}

Edizioni di Mary Astell, A Serious Proposal to the Ladies

A Serious Proposal to the Ladies, For the Advancement of their True and Greatest Interest. By a Lover of Her Sex. 1694. London: Printed for R. Wilkin at the King's Head in St. Paul's Church-Yard.

A Serious Proposal to the Ladies, For the Advancement of their True and Greatest Interest. By a Lover of Her Sex. 1695. London: Printed for R. Wilkin at the King's Head in St. Paul's Church-Yard [The Second Edition Corrected].

A Serious Proposal to the Ladies, For the Advancement of their True and Greatest Interest, part I By a Lover of Her Sex. 1696. London: Printed for R. Wilkin at the King's Head in St. Paul's Church-Yard [The Third Edition Corrected].

A Serious Proposal to the Ladies, For the Advancement of their True and Greatest Interest, part I By a Lover of Her Sex. 1701. London: Printed for R. Wilkin at the King's Head in St. Paul's Church-Yard [The Fourth Edition Corrected, nell'edizione del 1701 sono unite la parte I e II del Serious Proposal].

A Serious Proposal to the Ladies, part II. Wherein a Method as Offer'd for the Improvement of their Minds. 1697. London: Printed for R. Wilkin at the King's Head in St. Paul's Church-Yard.

A Serious Proposal to the Ladies, For the Advancement of their True and Greatest Interest, In Two Parts. By a Lover of Her Sex. 1697. London: Printed for R. Wilkin at the King's Head in St. Paul's Church-Yard.

Una seria proposta alle Signore. 1982. Roma: Lestoille [traduzione a cura di Rosmaria Loretelli].

A Serious Proposal to the Ladies. 1997. London: Pickering and Chatto [edited by Patricia Springborg].

\section{Fonti secondarie}

Allestre, Richard. 2016 [1673]. The Ladies Calling in Two Parts. Oxford: Oxford Theater.

Bochi, Giulia (a cura di). 1961. L'educazione femminile dall'umanesimo alla controriforma. Bologna: G. Malipiero. 
Cagnolati, Antonella. 2007. “Tra vita e finzione. Le donne e la scrittura nell'Europa del XVII secolo". Educazione al femminile. Una storia da scoprire, Ulivieri Simonetta (a cura di). Milano: Guerini. 97-115.

2016. La costilla de Ádan. Mujeres, educación y escritura en el Renacimiento. Sevilla: Benilde.

Cavendish, Margaret. 1655. The Worlds Olio. London: J. Martin and J. Allestrye.

Cleaver, Robert. 1610. A Godly Form of Householde Government. London: T. Man.

de Pizan, Christine. 1997. La città delle dame (Livre de la Cité des Dames). MilanoTrento: Luni Editrice.

Dillingham, Francis. 1609. Christian Oeconomy, or Household Government. London: J. Windet.

Erasmo da Rotterdam, 2002. Colloqui [a cura di C. Asso]. Torino: Einaudi.

Garin, Eugenio. (ed.). 1958. Il pensiero pedagogico dell'Umanesimo. Firenze: Giuntine Sansoni.

Gataker, Thomas. 1624. A Mariage Praier. London: F. Clifton.

Gouge, William. 1622. Of Domesticall Duties. London: J. Haviland.

Kelso, Ruth. 1978 [1956]. Doctrine for the Lady of the Renaissance. Urbana: University of Illinois Press.

Kelly-Gadol, Joan. 1987. "Did women have a Renaissance?” Becoming Visible. Women in European History. R. Bridenthal, C. Koonz, S. Stuard (Eds.). Boston: Houghton Mifflin. 175-201.

Kempe, William. 1588. The Education of Children in Learning. London: J. Parker and T. Gubbin.

Levin, Carole, The Heart and Stomach of a King: Elizabeth I and the Politics of Sex and Power, Philadelphia, University of Pennsylvania Press, 1994.

Maclean, Ian. 1980. The Renaissance Notion of Woman. Cambridge: Cambridge University Press.

Lenzi, Maria Ludovika. 1982. Donne e madonne. L'educazione femminile nel primo Rinascimento italiano. Torino: Loescher.

Mulcaster, Richard. 1581. Positions Wherein Those Primitive Circumstances Be Examined, Which Are Necessarie for the Training up of Children Either for Skill in Their Book or Health in Their Bodie. London: T. Chase.

Perry, Ruth. 1986. The Celebrated Mary Astell: An Early English Feminist. Chicago: University of Chicago Press. 
Purchas, Samuel. 1619. Purchas his Pilgrim: Microcosmus, or the Histoire of Man. London: W. Stansby.

Saville, George. 1688. Marchese di Halifax, The Lady'sNew-year'sGift: or, Advice to a Daughter. Under these following Heads, viz. Religion, Husband, House, Family and Children, Behaviour and Conversation, Friendships, Censure, Vanity and Affectation. Pride, Diversions. London: Matt Gillyflower and James Partridge.

Smith, Florence M. 1916. Mary Astell. New York: Columbia University Press.

Smith, Hilda. 1982. Reason's Disciples: Seventeenth-Century English Feminist. Urbana, Illinois: University of Illinois Press.

Thomas. K. V. 1958. "Women and the Civil War Sects", Past and Present, 13 (1958): 42-62.

Tertulliano. 1987. De cultu foeminarum (Gli ornamenti delle donne). Parma: Pratiche. 